\title{
REVIEW
}

Open Access

\section{Preclinical efficacy of stem cell therapy for skin flap: a systematic review and meta- analysis}

Yuan Li ${ }^{1}$, Qi-lin Jiang ${ }^{1}$, Leanne Van der Merwe ${ }^{2}$, Dong-hao Lou ${ }^{1}$ and Cai Lin ${ }^{1 *}$ (D)

\begin{abstract}
Background: A skin flap is one of the most critical surgical techniques for the restoration of cutaneous defects. However, the distal necrosis of the skin flap severely restricts the clinical application of flap surgery. As there is no consensus on the treatment methods to prevent distal necrosis of skin flaps, more effective and feasible interventions to prevent skin flaps from necrosis are urgently needed. Stem therapy as a potential method to improve the survival rate of skin flaps is receiving increasing attention.

Methods: This review followed the recommendations from the Preferred Reporting Items for Systematic Reviews and Meta-Analysis (PRISMA) statements. Twenty studies with 500 animals were included by searching Web of Science, EMBASE, PubMed, and Cochrane Library databases, up until October 8, 2020. Moreover, the references of the included articles were searched manually to obtain other studies. All analyses were conducted using Review Manager V.5.3 software.
\end{abstract}

Results: Meta-analysis of all 20 studies demonstrated stem cell treatment has significant effects on reducing necrosis of skin flap compared with the control group (SMD: 3.20, 95\% Cl 2.47 to 3.93). Besides, subgroup analysis showed differences in the efficacy of stem cells in improving the survival rate of skin flaps in areas of skin flap, cell type, transplant types, and method of administration of stem cells. The meta-analysis also showed that stem cell treatment had a significant effect on increasing blood vessel density (SMD: 2.96, 95\% Cl 2.21 to 3.72) and increasing the expression of vascular endothelial growth factor (VEGF, SMD: 4.34, 95\% Cl 2.48 to 6.1).

Conclusions: The preclinical evidence of our systematic review indicate that stem cell-based therapy is effective for promoting early angiogenesis by up regulating VEGF and ultimately improving the survival rate of skin flap. In summary, small area skin flap, the administration method of intra-arterial injection, ASCs and MSCs, and xenogenic stem cells from humans showed more effective for the survival of animal skin flaps. In general, stem cell-based therapy may be a promising method to prevent skin flap necrosis.

Keywords: Meta-analysis, Preclinical evidence, Skin flaps, Stem cell

\footnotetext{
* Correspondence: 13025092850@163.com

'Department of Burn, The First Affiliated Hospital of Wenzhou Medical

University, Nan Bai Xiang, Wenzhou, Zhejiang 325000, People's Republic of

China

Full list of author information is available at the end of the article
}

(C) The Author(s). 2021 Open Access This article is licensed under a Creative Commons Attribution 4.0 International License, which permits use, sharing, adaptation, distribution and reproduction in any medium or format, as long as you give appropriate credit to the original author(s) and the source, provide a link to the Creative Commons licence, and indicate if changes were made. The images or other third party material in this article are included in the article's Creative Commons licence, unless indicated otherwise in a credit line to the material. If material is not included in the article's Creative Commons licence and your intended use is not permitted by statutory regulation or exceeds the permitted use, you will need to obtain permission directly from the copyright holder. To view a copy of this licence, visit http://creativecommons.org/licenses/by/4.0/ The Creative Commons Public Domain Dedication waiver (http://creativecommons.org/publicdomain/zero/1.0/) applies to the data made available in this article, unless otherwise stated in a credit line to the data. 


\section{Introduction}

A skin flap is one of the most critical surgical techniques for the restoration of cutaneous defects caused by trauma, tumor excision, lower limb vascular ulcer, or diabetes mellitus [1-3]. However, for skin flaps, especially for the treatment of large areas, distal necrosis is one of the most common postoperative complications [4]. This complication makes the ratio of length to width of the flap to be $1.5-2$, which severely restricts the clinical application of flap surgery [5]. Clinical experience has shown that once the skin flap becomes necrotic, it will not only lead to the increase of possible secondary surgery and treatment costs, but also more pain and suffering [6]. Further, the main mechanisms of flap necrosis are insufficient blood perfusion, venous return disorder, and ischemiareperfusion injury. If the degree of ischemia exceeds the tolerance threshold of the tissue without intervention in a short period of time, the ischemic part of the tissue will undergo irreversible necrosis [7]. To prevent necrosis of the skin flap, it is the key to improve local neovascularization and increase the blood supply to ischemic tissues. Therefore, appropriate exogenous intervention is necessary to accelerate early angiogenesis to prevent postoperative necrosis of the flap [8]. To make more flaps survive successfully, various strategies for preventing skin flap necrosis have recently been developed, including reduction of oxidative stress [9], inhibition of apoptosis [10], and vasodilators [11]. However, due to the bad effects of all the above mentioned treatment methods, there is no consensus on the treatment methods to prevent distal necrosis of skin flaps. Therefore, more effective and feasible interventions to prevent skin flaps from necrotizing are urgently needed.

Angiogenesis in skin flaps is an intricate process involving the coordination of various cells and cytokines [12]. Stem cells have the unique ability of self-renewal and differentiation into different cells, which provides a new possibility for regenerative medicine [13, 14]. Furthermore, many studies have revealed that stem cell therapy has a significant effect in protecting the heart, brain, and kidneys from ischemic damage and improving prognosis [15-17]. With this in mind, cell therapy as a potential method to improve the survival rate of skin flaps is receiving increasing attention $[8,18,19]$. However, even many animal experiments focus on this topic; unfortunately, there is almost no research on its clinical efficacy, let alone become the gold standard for clinical treatment of skin flaps. What is more, animal experiments, a bridge between the bench and the bedside, help assess the efficacy of stem cells and clarify further mechanisms [20]. Even many studies have hitherto explored the role of stem cells for skin flaps in animals, their efficacy and mechanisms have not been systematically summarized. To ensure that this promising and evidence- based stem cell therapy may develop into future clinical practice for patients who need skin flap surgery, we have conducted a systematic review of those animal studies to investigate the efficacy of stem cells for skin flaps.

\section{Methods}

This study followed the recommendations from the Preferred Reporting Items for Systematic Reviews and Meta-Analysis (PRISMA) statements [21]. The PRISMA 2009 checklist was shown in Supplementary Table 1. Our protocol was published through PROSPERO (CRD42020213388) and also can be found online at https://www.crd.york.ac.uk/prospero/display_record.

php? RecordID=213388z.

\section{Search strategy}

Multiple databases (including PubMed, Web of Science, EMBASE, and Cochrane libraries) have been searched for related studies on improving the survival rate of skin flaps by stem cell therapy, up until October 8, 2020. We selected the following terms: (1) "mesenchymal stem cell" OR "progenitor cells" OR "mononuclear cells" OR "stem cell(s)" OR "mesenchymal stem cell" OR "Mesenchymal Stromal Cells" OR "adipose-derived stem cells" AND (2) "skin flap" OR "skin flaps". All articles are limited to preclinical studies and published in English. Moreover, the references of the included articles were searched manually to obtain other studies.

\section{Eligibility criteria}

The selection criteria for this study were prespecified as follows: (1) published as an original research article, (2) experimental models of skin flaps, (3) treatment groups treated with stem cells, (4) control group only received the liquid without therapeutic effect or no treatment, and (5) the primary outcome was the survival rate of skin flaps. The second outcome measures were blood vessel density and expression of vascular endothelial growth factor (VEGF). The exclusion criteria of studies were prespecified as follows: (1) animal models unrelated to skin flaps, (2) the application of stem cell in combination with other treatment methods in the treatment group, (3) no control group, and (4) clinical trial, review article, and duplicate publication.

\section{Data extraction}

The studies that did not meet the inclusion criteria were excluded after screening all the articles searched. Two authors read the full text independently and extracted the relevant data. The differences raised during this period were handled and resolved by a third author. The following details were recorded: (1) the first author, year, and country of studies; (2) the species and number of animals, and the area of the flap; (3) origin, type, and 
quantity of stem cells; (4) intervention measures of treatment group and control group; and (5) primary and secondary outcome measurements collected. When some data was presented only in the form of figures, we tried to contact the author for more detailed data. If we did not get the corresponding reply from the author, we used the digital ruler software to measure the pictures to obtain the data.

\section{Assessment of the risk of bias}

The quality assessment was carried out by two research experts independently, and the possible differences were comprehensively evaluated according to the opinions of the third expert. The risk of bias in our animal studies was assessed by a minor modified 10 -item scale. The following domains were assessed: (a) sequence generation, (b) baseline characteristics, (c) allocation concealment, (d) random housing, (e) blinding of investigators, (f) random outcome assessment, ( $\mathrm{g}$ ) blinding of outcome assessor, (h) complete outcome data, (i) selective outcome reporting, and (j) other sources of bias.

\section{Statistical analysis}

All analyses were conducted using the Review Manager V.5.3 software. Outcomes were continuous data and presented as standardized mean difference (SMD) with 95\% confidence interval when the scales of data are inconsistent. The results of the meta-analysis are presented with forest diagram. Heterogeneity and choice of effects models were probed with the Cochrane Qstatistic test and the $I^{2}$-statistic test. When $I^{2}>50 \%$, indicating that the included studies have significant heterogeneity, and a random-effect meta-analysis model is used. Instead, a fixed-effect model was adopted. Explore the source of the heterogeneity when inter-study heterogeneity was obvious, and sensitivity analysis or subgroup analysis was conducted if necessary. Funnel plots were drawn to intuitively investigate publication bias when there were no less than ten studies that reported the same outcome measurement.

\section{Results}

\section{Study selection}

Our last search was conducted on October 1, 2020 (Fig. 1). A total of 53 potential hints were obtained in the initial search through the database. After excluding 22 irrelevant or reduplicated studies, the remaining 31 studies were further screened. After a screening of the title and abstract of the remaining studies and a careful reading of the full text, 11 studies were excluded for the

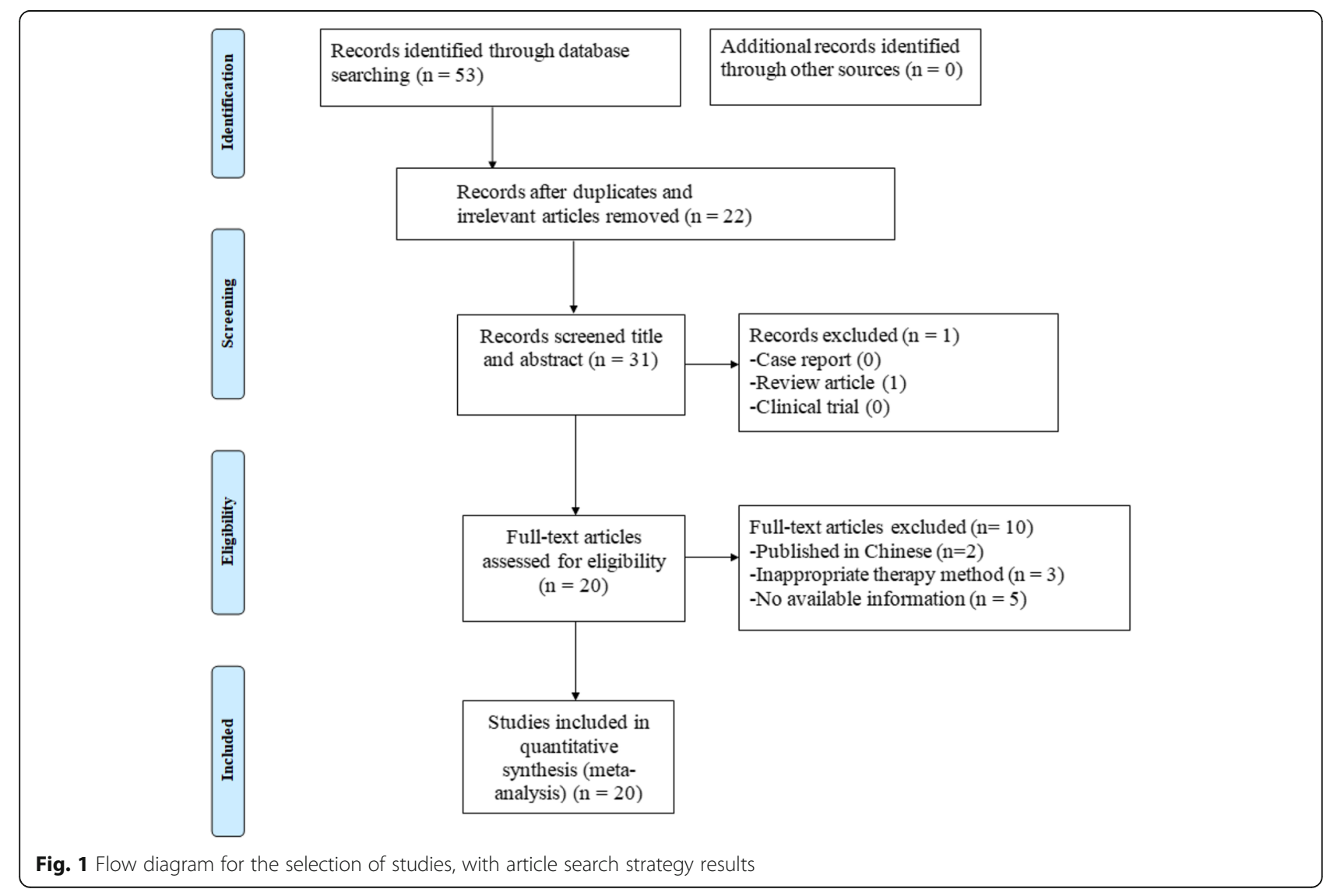


following reasons: (1) review article [22], (2) published in Chinese $[23,24],(3)$ inappropriate therapy method [2527], and (4) no available information [28-32]. Finally, twenty studies were included, and a meta-analysis of stem cells for skin flaps was conducted $[8,18,19,28$, $33-48]$.

\section{Characteristics of included studies}

A total of 20 studies and 24 treatment groups were included, and further data extraction was performed according to the classification listed in Table 1 . These studies were published between 2010 and 2020. In this meta-analysis, a total of nine studies were completed in China $[18,19,33,34,40,41,43-45]$, four in Iran $[8,46-$ 48], four in Korea [28, 38, 39, 42], and the remaining three in Germany [37], Brazil [36], and the USA [36]. For animal selection, $60 \%$ of the experiments used mice as models $[8,28,33,34,36,37,41,42,45-48]$, and $40 \%$ of the studies used rats [18, 19, 35, 38-40, 43, 44]. Most studies used random skin flap, and only three studies used axial skin flap [37, 40, 45]. The area of the skin flaps varies from 1.25 to $40 \mathrm{~cm}^{2}$ in the selected studies. Cell types were also compared, such that 11 of the included studies used MSCs, nine used ASCs, and one used MNCs [33]. It is worth mentioning that one study used both adipose-derived stem cells and mononuclear cells [33]. In terms of transplant type, 12 studies used allogenic cells [8, 33-37, 39, 42, 45-48], and eight studies used xenogenic cells $[18,19,28,38,40,41,43,44]$. The number of cells given ranged from $10^{3}$ to $6 \times 10^{9}$. The mode of administration varied by study, such that $75 \%$ of 24 treatment arms used subcutaneous injection, $12.5 \%$ intra-arterial injection, and $12.5 \%$ intravenous injection. The intervention measures in the control group were mainly phosphate-buffered saline (PBS) [36, 38, 44, 45], medium [19, 33, 34, 37, 40, 42, 43], saline [8, 46, $47]$, and no treatment $[18,28,35,39,41,48]$. For outcome measures, survival rate of the flap was used in all studies, blood vessel density in ten studies $[18,19,28$, 34, 37, 39, 40, 43, 44, 48], and expression of vascular endothelial growth factor (VEGF) in six studies $[8,33$, $34,40,43,44]$.

\section{Quality of included studies}

As evaluated by a modified 10-item scale, the 20 included studies were medium-quality animal experiments. The risk of biases for all the included studies is shown in Table 2. Few studies did not use random allocation [19, $28,35,45]$, and all studies reported baseline characteristics but did not allocation concealment. It should be noted that random housing is not mentioned in all studies. Under the domain for "blinding of investigators," two studies were assessed low risk of bias [37, 44], while the rest of the studies were considered as unclear risk of bias. Seven studies described the use of random outcome for assessment [33, 34, 39-42, 44], and eight studies conducted a blind method to outcome assessor [18, 37, $39,40,42-44,48]$. In our meta-analysis, all studies are considered to report the outcome data completely and avoid outcome. Notably, there was uncertainty regarding the other sources of bias.

\section{Effect size \\ Primary outcome measures}

Survival rate of flap Survival rate of flap, the most obvious result data, is used as the primary outcome measure in this systematic review. Meta-analysis of all 20 studies demonstrated stem cell treatment has significant effects on reducing necrosis of skin flap compared with control group $(n=480$, SMD $3.20,95 \%$ CI 2.47 to $3.93, P<$ $0.00001 ; I^{2}=84 \%$, Fig. 2).

Since significant heterogeneity was found in this metaanalysis, we conducted further analysis of the source of heterogeneity, including sensitivity and subgroup analysis. However, the results of the sensitivity analysis showed that the heterogeneity did not decrease significantly after excluding individual studies in turn. Thus, subgroup analysis was performed by grouping studies according to the following classification: type of skin flap, area of skin flap, cell type, cell number, transplant types, and method of administration of stem cells used in treatment. The dose of stem cells in the intervention groups and the area of skin flaps varied widely in the included studies. Therefore, we divided the dose of stem cells into low $\left(<5 \times 10^{6}\right.$ cells $)$ and high $\left(\geq 5 \times 10^{6}\right.$ cells $)$ in advance and divided the area of skin flaps into small $\left(<10 \mathrm{~cm}^{2}\right)$ and large $\left(\geq 10 \mathrm{~cm}^{2}\right)$. The results of the subgroup analysis showed that there was no obvious heterogeneity between the subgroups in terms of flap types (Fig. S1 Supplementary materials), stem cell number (Fig. S2 Supplementary materials), and treatment measures of the control group (Fig. S3 Supplementary materials). Notably, although all types have been proven to be effective to skin flaps, adipose-derived stem cells (ASCs; SMD 3.39) and mesenchymal stem cells (MSCs; SMD 3.29) are considered to show a statistically larger effect size than mononuclear cells (MNCs; SMD 2.07; Fig. S4 Supplementary materials). In the subgroup analysis of skin flap area, both small area $\left(<10 \mathrm{~cm}^{2}\right)$ and large area $\left(\geq 10 \mathrm{~cm}^{2}\right)$ demonstrated the effect of stem cell therapy on skin flaps, with the former being more effective (SMD 3.88 vs 2.79; Fig. S5 Supplementary materials). Interestingly, in terms of skin flap survival rate, the therapeutic effect of xenogeneic stem cells is significantly better than that of allogeneic transplantation types (SMD 4.51 vs 2.35; Fig. S6 Supplementary materials). By comparing the studies of different administration routes, 
Table 1 Characteristics of the included studies

\begin{tabular}{|c|c|c|c|c|c|c|c|c|c|c|c|}
\hline Study (year) & Country & $\begin{array}{l}\text { Animal } \\
\text { (number) }\end{array}$ & $\begin{array}{l}\text { Type of } \\
\text { skin } \\
\text { flap }\end{array}$ & $\begin{array}{l}\text { Skin } \\
\text { flap } \\
\left(\mathrm{cm}^{2}\right)\end{array}$ & $\begin{array}{l}\text { Cell } \\
\text { type }\end{array}$ & $\begin{array}{l}\text { Tissue of } \\
\text { origin }\end{array}$ & $\begin{array}{l}\text { Transplant } \\
\text { type }\end{array}$ & $\begin{array}{l}\text { Cell } \\
\text { number }\end{array}$ & $\begin{array}{l}\text { Method of } \\
\text { administration }\end{array}$ & Placebo & Outcome index \\
\hline $\begin{array}{l}\text { Chehelcheraghi } \\
\text { et al., } 2020 \text { [48] }\end{array}$ & Iran & $\begin{array}{l}\text { Wistar } \\
\text { rats (10) }\end{array}$ & Random & 24 & MSCS & $\begin{array}{l}\text { Bone } \\
\text { marrow }\end{array}$ & Allogenic & $6 \times 10^{9}$ & $\begin{array}{l}\text { Subcutaneous } \\
\text { injection }\end{array}$ & None & $\begin{array}{l}\text { 1. Survival rate of } \\
\text { flap 2. Blood vessel } \\
\text { density }\end{array}$ \\
\hline $\begin{array}{l}\text { Chehelcheraghi } \\
\text { et al., } 2019 \text { [8] }\end{array}$ & Iran & $\begin{array}{l}\text { Wistar } \\
\text { rats (20) }\end{array}$ & Random & 24 & MSCs & $\begin{array}{l}\text { Bone } \\
\text { marrow }\end{array}$ & Allogenic & $6 \times 10^{9}$ & $\begin{array}{l}\text { Subcutaneous } \\
\text { injection }\end{array}$ & Saline & $\begin{array}{l}\text { 1. Survival rate of } \\
\text { flap 2. VEGF }\end{array}$ \\
\hline $\begin{array}{l}\text { Chehelcheraghi } \\
\text { et al., } 2016 \text { [47] }\end{array}$ & Iran & $\begin{array}{l}\text { Wistar } \\
\text { rats (20) }\end{array}$ & Random & 24 & MSCs & $\begin{array}{l}\text { Bone } \\
\text { marrow }\end{array}$ & Allogenic & $1 \times 10^{9}$ & $\begin{array}{l}\text { Subcutaneous } \\
\text { injection }\end{array}$ & Saline & $\begin{array}{l}\text { 1. Survival rate of } \\
\text { flap }\end{array}$ \\
\hline $\begin{array}{l}\text { Chehelcheraghi } \\
\text { et al., } 2015 \text { [46] }\end{array}$ & Iran & $\begin{array}{l}\text { Wistar } \\
\text { rats (20) }\end{array}$ & Random & 24 & MSCS & $\begin{array}{l}\text { Bone } \\
\text { marrow }\end{array}$ & Allogenic & $1 \times 10^{9}$ & $\begin{array}{l}\text { Subcutaneous } \\
\text { injection }\end{array}$ & Saline & $\begin{array}{l}\text { 1. Survival rate of } \\
\text { flap }\end{array}$ \\
\hline $\begin{array}{l}\text { Ding et al. \#1, } \\
2020 \text { [45] }\end{array}$ & China & $\begin{array}{l}\text { Wistar } \\
\text { rats (12) }\end{array}$ & Axial & 25 & MSCs & $\begin{array}{l}\text { Bone } \\
\text { marrow }\end{array}$ & Allogenic & $1 \times 10^{6}$ & $\begin{array}{l}\text { Subcutaneous } \\
\text { injection }\end{array}$ & PBS & $\begin{array}{l}\text { 1. Survival rate of } \\
\text { flap }\end{array}$ \\
\hline $\begin{array}{l}\text { Ding et al. \#2, } \\
2020 \text { [45] }\end{array}$ & China & $\begin{array}{l}\text { Wistar } \\
\text { rats (12) }\end{array}$ & Axial & 25 & MSCs & $\begin{array}{l}\text { Bone } \\
\text { marrow }\end{array}$ & Allogenic & $5 \times 10^{6}$ & $\begin{array}{l}\text { Subcutaneous } \\
\text { injection }\end{array}$ & PBS & $\begin{array}{l}\text { 1. Survival rate of } \\
\text { flap }\end{array}$ \\
\hline $\begin{array}{l}\text { Feng et al. \#1, } \\
2020 \text { [44] }\end{array}$ & China & $\begin{array}{l}\text { BALB/C } \\
\text { mice }(20)\end{array}$ & Random & 9 & ASCs & Adipose & Xenogenic & $1 \times 10^{3}$ & $\begin{array}{l}\text { Intra-arterial } \\
\text { injection }\end{array}$ & PBS & $\begin{array}{l}\text { 1. Survival rate of } \\
\text { flap 2. Blood vessel } \\
\text { density 3. VEGF }\end{array}$ \\
\hline $\begin{array}{l}\text { Feng et al. \#2, } \\
2020 \text { [44] }\end{array}$ & China & $\begin{array}{l}\text { BALB/C } \\
\text { mice }(20)\end{array}$ & Random & 9 & ASCs & Adipose & Xenogenic & $1 \times 10^{4}$ & $\begin{array}{l}\text { Intra-arterial } \\
\text { injection }\end{array}$ & PBS & $\begin{array}{l}\text { 1. Survival rate of } \\
\text { flap 2. Blood vessel } \\
\text { density 3. VEGF }\end{array}$ \\
\hline $\begin{array}{l}\text { Feng et al. \#3, } \\
2020 \text { [44] }\end{array}$ & China & $\begin{array}{l}\text { BALB/C } \\
\text { mice }(20)\end{array}$ & Random & 9 & ASCs & Adipose & Xenogenic & $1 \times 10^{5}$ & $\begin{array}{l}\text { Intra-arterial } \\
\text { injection }\end{array}$ & PBS & $\begin{array}{l}\text { 1. Survival rate of } \\
\text { flap 2. Blood vessel } \\
\text { density 3. VEGF }\end{array}$ \\
\hline $\begin{array}{l}\text { Gao et al., } 2011 \\
\text { [43] }\end{array}$ & China & $\begin{array}{l}\text { BALB/C } \\
\text { mice }(30)\end{array}$ & Random & 3 & ASCs & Adipose & Xenogenic & $1 \times 10^{7}$ & $\begin{array}{l}\text { Subcutaneous } \\
\text { injection }\end{array}$ & Medium & $\begin{array}{l}\text { 1. Survival rate of } \\
\text { flap 2. Blood vessel } \\
\text { density 3. VEGF }\end{array}$ \\
\hline $\begin{array}{l}\text { Han et al., } 2015 \\
{[42]}\end{array}$ & Korea & $\begin{array}{l}\text { SD rats } \\
(14)\end{array}$ & Random & 24 & ASCs & Adipose & Allogenic & $5 \times 10^{5}$ & $\begin{array}{l}\text { Subcutaneous } \\
\text { injection }\end{array}$ & Medium & $\begin{array}{l}\text { 1. Survival rate of } \\
\text { flap }\end{array}$ \\
\hline $\begin{array}{l}\text { Leng et al., } \\
2017 \text { [41] }\end{array}$ & China & $\begin{array}{l}\text { Wistar } \\
\text { rats (48) }\end{array}$ & Random & 18 & MSCs & $\begin{array}{l}\text { Umbilical } \\
\text { cord }\end{array}$ & Xenogenic & $4 \times 10^{5}$ & $\begin{array}{l}\text { Subcutaneous } \\
\text { injection }\end{array}$ & None & $\begin{array}{l}\text { 1. Survival rate of } \\
\text { flap }\end{array}$ \\
\hline $\begin{array}{l}\text { Leng et al., } \\
2012 \text { [40] }\end{array}$ & China & $\begin{array}{l}\mathrm{BALB} / \mathrm{C} \\
\text { mice }(20)\end{array}$ & Axial & 18 & MSCS & $\begin{array}{l}\text { Umbilical } \\
\text { cord }\end{array}$ & Xenogenic & $4 \times 10^{5}$ & $\begin{array}{l}\text { Subcutaneous } \\
\text { injection }\end{array}$ & Medium & $\begin{array}{l}\text { 1. Survival rate of } \\
\text { flap 2. Blood vessel } \\
\text { density 3. VEGF }\end{array}$ \\
\hline $\begin{array}{l}\text { Moon et al., } \\
2018 \text { [39] }\end{array}$ & Korea & $\begin{array}{l}\text { ICR mice } \\
(16)\end{array}$ & Random & 4.5 & MSCS & $\begin{array}{l}\text { Bone } \\
\text { marrow }\end{array}$ & Allogenic & $2 \times 10^{6}$ & $\begin{array}{l}\text { Subcutaneous } \\
\text { injection }\end{array}$ & None & $\begin{array}{l}\text { 1. Survival rate of } \\
\text { flap 2. Blood vessel } \\
\text { density }\end{array}$ \\
\hline $\begin{array}{l}\text { Pak et al., } 2020 \\
\text { [28] }\end{array}$ & Korea & $\begin{array}{l}\text { SD rats } \\
(12)\end{array}$ & Random & 27 & ASCs & Adipose & Xenogenic & $5 \times 10^{6}$ & $\begin{array}{l}\text { Subcutaneous } \\
\text { injection }\end{array}$ & None & $\begin{array}{l}\text { 1. Survival rate of } \\
\text { flap 2. Blood vessel } \\
\text { density }\end{array}$ \\
\hline $\begin{array}{l}\text { Park et al., } 2017 \\
\text { [38] }\end{array}$ & Korea & $\begin{array}{l}\text { BALB/C } \\
\text { mice (16) }\end{array}$ & Random & 8 & ASCs & Adipose & Xenogenic & $\begin{array}{l}1.5 \times \\
10^{6}\end{array}$ & $\begin{array}{l}\text { Subcutaneous } \\
\text { injection }\end{array}$ & PBS & $\begin{array}{l}\text { 1. Survival rate of } \\
\text { flap }\end{array}$ \\
\hline $\begin{array}{l}\text { Pu et al., } 2017 \\
{[19]}\end{array}$ & China & $\begin{array}{l}\text { C57BL/6 J } \\
\text { mice (12) }\end{array}$ & Random & 4 & ASCs & Adipose & Xenogenic & $1 \times 10^{6}$ & $\begin{array}{l}\text { Subcutaneous } \\
\text { injection }\end{array}$ & Medium & $\begin{array}{l}\text { 1. Survival rate of } \\
\text { flap 2. Blood vessel } \\
\text { density }\end{array}$ \\
\hline $\begin{array}{l}\text { Reichenberger } \\
\text { et al., } 2012 \text { [37] }\end{array}$ & Germany & $\begin{array}{l}\text { Lewis rats } \\
\text { (16) }\end{array}$ & Axial & 60 & ASCs & Adipose & Allogenic & $5 \times 10^{6}$ & $\begin{array}{l}\text { Intravenous } \\
\text { injection }\end{array}$ & Medium & $\begin{array}{l}\text { 1. Survival rate of } \\
\text { flap 2. Blood vessel } \\
\text { density }\end{array}$ \\
\hline $\begin{array}{l}\text { Suartz et al., } \\
2014[36]\end{array}$ & Brazil & $\begin{array}{l}\text { Wistar } \\
\text { rats (30) }\end{array}$ & Random & 40 & ASCs & Adipose & Allogenic & $5 \times 10^{6}$ & $\begin{array}{l}\text { Intravenous } \\
\text { injection }\end{array}$ & PBS & $\begin{array}{l}\text { 1. Survival rate of } \\
\text { flap 2. Blood vessel } \\
\text { density }\end{array}$ \\
\hline $\begin{array}{l}\text { Tang et al., } \\
2016 \text { [35] }\end{array}$ & USA & $\begin{array}{l}\text { C57Bl6 } \\
\text { mice (12) }\end{array}$ & Random & 2 & MSCs & $\begin{array}{l}\text { Bone } \\
\text { marrow }\end{array}$ & Allogenic & $3 \times 10^{6}$ & $\begin{array}{l}\text { Intravenous } \\
\text { injection }\end{array}$ & None & $\begin{array}{l}\text { 1. Survival rate of } \\
\text { flap 2. Blood vessel } \\
\text { density }\end{array}$ \\
\hline $\begin{array}{l}\text { Wang et al., } \\
2011 \text { [34] }\end{array}$ & China & $\begin{array}{l}\text { SD rats } \\
(20)\end{array}$ & Random & 16 & MSCs & $\begin{array}{l}\text { Bone } \\
\text { marrow }\end{array}$ & Allogenic & $4 \times 10^{6}$ & $\begin{array}{l}\text { Subcutaneous } \\
\text { injection }\end{array}$ & Medium & $\begin{array}{l}\text { 1. Survival rate of } \\
\text { flap 2. Blood vessel } \\
\text { density 3. VEGF }\end{array}$ \\
\hline
\end{tabular}


Table 1 Characteristics of the included studies (Continued)

\begin{tabular}{|c|c|c|c|c|c|c|c|c|c|c|c|}
\hline Study (year) & Country & $\begin{array}{l}\text { Animal } \\
\text { (number) }\end{array}$ & $\begin{array}{l}\text { Type of } \\
\text { skin } \\
\text { flap }\end{array}$ & $\begin{array}{l}\text { Skin } \\
\text { flap } \\
\left(\mathrm{cm}^{2}\right)\end{array}$ & $\begin{array}{l}\text { Cell } \\
\text { type }\end{array}$ & $\begin{array}{l}\text { Tissue of } \\
\text { origin }\end{array}$ & $\begin{array}{l}\text { Transplant } \\
\text { type }\end{array}$ & $\begin{array}{l}\text { Cell } \\
\text { number }\end{array}$ & $\begin{array}{l}\text { Method of } \\
\text { administration }\end{array}$ & Placebo & Outcome index \\
\hline $\begin{array}{l}\text { Yang et al. \#1, } \\
2010 \text { [33] }\end{array}$ & China & $\begin{array}{l}\text { Wistar } \\
\text { rats (20) }\end{array}$ & Random & 27 & MNCs & $\begin{array}{l}\text { Bone } \\
\text { marrow }\end{array}$ & Allogenic & $1 \times 10^{8}$ & $\begin{array}{l}\text { Subcutaneous } \\
\text { injection }\end{array}$ & Medium & $\begin{array}{l}\text { 1. Survival rate of } \\
\text { flap 2. VEGF }\end{array}$ \\
\hline $\begin{array}{l}\text { Yang et al. \#2, } \\
2010 \text { [33] }\end{array}$ & China & $\begin{array}{l}\text { Wistar } \\
\text { rats (20) }\end{array}$ & Random & 27 & ASCs & Adipose & Allogenic & $4 \times 10^{6}$ & $\begin{array}{l}\text { Subcutaneous } \\
\text { injection }\end{array}$ & Medium & $\begin{array}{l}\text { 1. Survival rate of } \\
\text { flap 2. VEGF }\end{array}$ \\
\hline $\begin{array}{l}\text { Zhou et al., } \\
2019 \text { [18] }\end{array}$ & China & $\begin{array}{l}\text { BALB/C } \\
\text { mice }(40)\end{array}$ & Random & 1.25 & MSCs & $\begin{array}{l}\text { Bone } \\
\text { marrow }\end{array}$ & Xenogenic & $\begin{array}{l}2.5 \times \\
10^{4}\end{array}$ & $\begin{array}{l}\text { Subcutaneous } \\
\text { transplantation }\end{array}$ & None & $\begin{array}{l}\text { 1. Survival rate of } \\
\text { flap 2. Blood vessel } \\
\text { density }\end{array}$ \\
\hline
\end{tabular}

ASCs adipose-derived stem cells, ICR imprinting control region, MNCs mononuclear cells, MSCs mesenchymal stem cells, NA not available, $P B S$ phosphate-buffered saline, SD Sprague-Dawley, VEGF vascular endothelial growth factor

we discovered that intravascular injection, including intra-arterial injection (SMD 5.45, 95\% CI 2.25 to 8.64 ) and intravenous injection (SMD 3.86, 95\% CI 2.92 to 4.80), has a more significant effect in preventing skin flap necrosis, compared to subcutaneous injection (SMD 2.77, 95\% CI 1.96 to 3.58; Fig. S7 Supplementary materials). However, this result may be affected by other factors. For example, only a few studies [35-37, 44] have used the method of administration of intravascular injection.

Table 2 Risk of bias of the included studies

\begin{tabular}{|c|c|c|c|c|c|c|c|c|c|c|c|}
\hline Study & A & B & $\mathrm{C}$ & D & $E$ & $\mathbf{F}$ & G & $\mathrm{H}$ & I & $J$ & Toal \\
\hline Chehelcheraghi et al., 2020 [48] & + & + & - & $?$ & $?$ & $?$ & + & + & + & $?$ & 5 \\
\hline Chehelcheraghi et al., 2019 [8] & + & + & - & $?$ & $?$ & $?$ & $?$ & + & + & $?$ & 4 \\
\hline Chehelcheraghi et al., 2016 [47] & + & + & - & $?$ & $?$ & $?$ & $?$ & + & + & $?$ & 4 \\
\hline Chehelcheraghi et al., 2015 [46] & + & + & - & $?$ & $?$ & $?$ & $?$ & + & + & $?$ & 4 \\
\hline Ding et al., 2020 [45] & $?$ & + & - & $?$ & $?$ & $?$ & $?$ & + & + & $?$ & 3 \\
\hline Feng et al., 2020 [44] & + & + & - & $?$ & + & + & + & + & + & $?$ & 7 \\
\hline Gao et al., 2011 [43] & + & + & - & $?$ & $?$ & $?$ & + & + & + & $?$ & 5 \\
\hline Han et al., 2015 [42] & + & + & - & $?$ & $?$ & + & + & + & + & $?$ & 6 \\
\hline Leng et al., 2017 [41] & + & + & - & $?$ & $?$ & + & $?$ & + & + & $?$ & 5 \\
\hline Leng et al., 2012 [40] & + & + & - & $?$ & $?$ & + & + & + & + & $?$ & 6 \\
\hline Moon, 2018 [39] & + & + & - & $?$ & $?$ & + & + & + & + & $?$ & 6 \\
\hline Pak et al., 2020 [28] & $?$ & + & - & $?$ & $?$ & $?$ & $?$ & + & + & $?$ & 3 \\
\hline Park et al., 2017 [38] & + & + & - & $?$ & $?$ & $?$ & $?$ & + & + & $?$ & 4 \\
\hline Pu et al., 2017 [19] & $?$ & + & - & $?$ & $?$ & $?$ & $?$ & + & + & $?$ & 3 \\
\hline Reichenberger et al., 2012 [37] & + & + & - & $?$ & + & $?$ & + & + & + & $?$ & 5 \\
\hline Suartz et al., 2014 [36] & + & + & - & $?$ & $?$ & $?$ & $?$ & + & + & $?$ & 4 \\
\hline Tang et al., 2016 [35] & $?$ & + & - & $?$ & $?$ & $?$ & $?$ & + & + & $?$ & 3 \\
\hline Wang et al., 2011 [34] & + & + & - & $?$ & $?$ & + & $?$ & + & + & $?$ & 5 \\
\hline Yang et al., 2010 [33] & + & + & - & $?$ & $?$ & + & $?$ & + & + & $?$ & 5 \\
\hline Zhou et al., 2019 [18] & + & + & - & $?$ & $?$ & $?$ & + & + & + & $?$ & 5 \\
\hline
\end{tabular}

Studies fulfilling the criteria of $A$ sequence generation, $B$ baseline characteristics, $C$ allocation concealment, $D$ random housing, $E$ blinding of investigators, $F$ random outcome assessment, $G$ blinding of outcome assessor, $H$ complete outcome data, I selective outcome reporting, and $J$ other sources of bias

\section{Secondary outcome measures}

Blood vessel density The meta-analysis of 12 groups demonstrated stem cell treatment has significant effects on increasing blood vessel density compared with control group ( $n=236$, SMD 2.96, 95\% CI 2.21 to $3.72, P<$ $0.00001 ; I^{2}=70 \%$, Fig. 3).

VEGF Meta-analysis of nine groups demonstrated stem cell-treated group was superior to the control group according to the increased the expression of VEGF $(n=$ 190, SMD 4.34, 95\% CI 2.48 to $6.19, P<0.00001 ; I^{2}=$ 93\%, Fig. 4).

\section{Publication bias}

We evaluated publication bias of survival rate of skin flaps and blood vessel density by funnel plots. As shown in Fig. 5, the trend of the funnel plot of flap survival rate and blood vessel density is generally the same. The distribution of the funnel plots was slightly asymmetric, indicating that there may be potential publication bias.

\section{Discussion}

As we all know, a free skin flap or pedicled skin flap transplantation is an indispensable key technology in numerous surgical fields to repair large tissue defects [49, 50]. Part of or all of skin flap necrosis is a common postoperative complication, and tissue ischemia caused by insufficient blood supply often leads to skin flap failure [51]. However, stem cells have the ability of self-renewal and differentiation into various cell lines, which provides the possibility of early angiogenesis of the skin flap [19, 52]. Therefore, the purpose of this review is to provide preclinical evidence for the effectiveness of stem cell therapy for skin flaps.

To our knowledge, this is the first preclinical systematic review and meta-analysis to estimate the efficacy and possible mechanism of stem cell therapy in promoting flap survival. The study is timely, considering that various stem cell therapeutic potentials are currently being tested in numerous preclinical trials, and clinical 


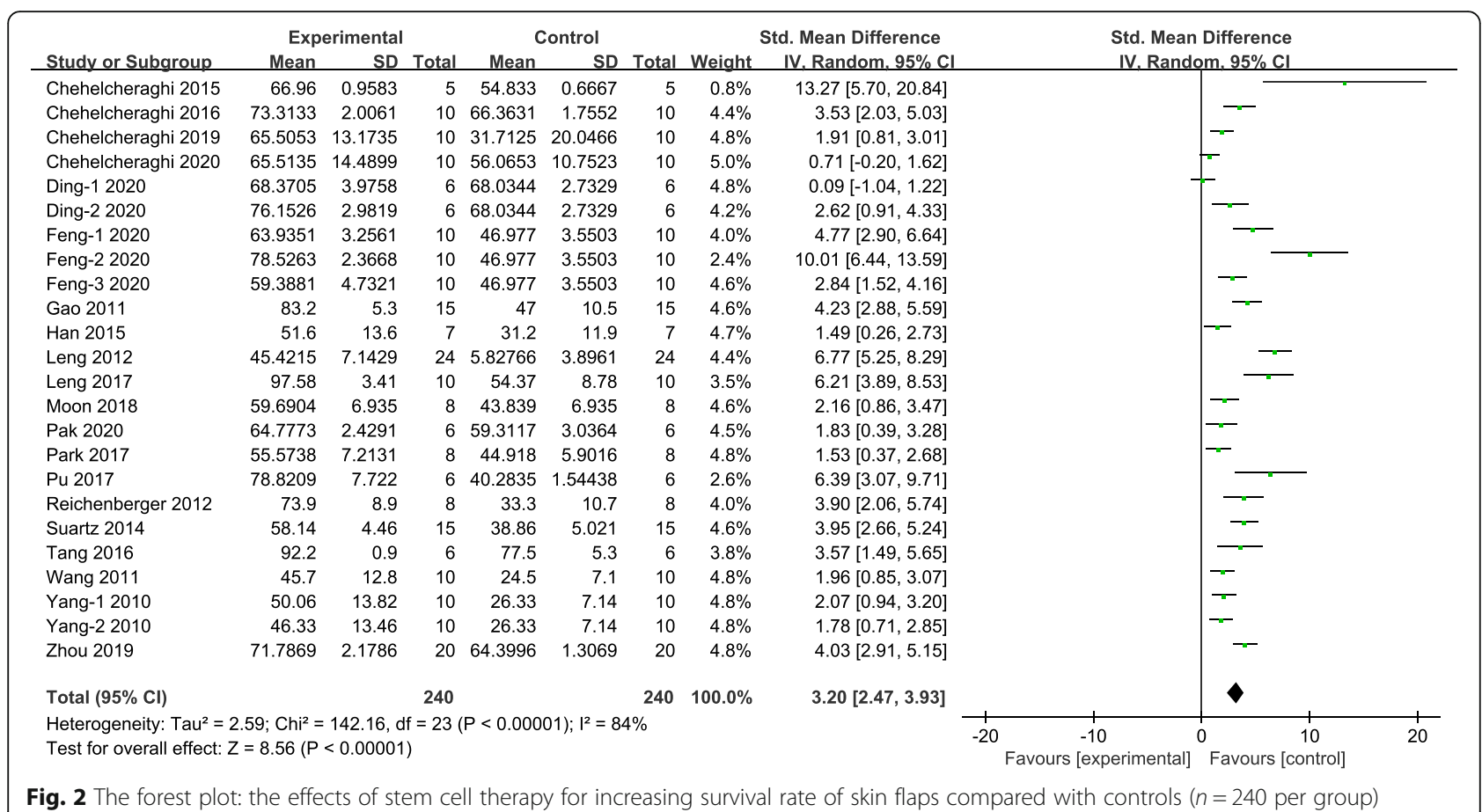

trials are almost not conducted. Our meta-analysis included 20 studies with 500 animals and analyzed three outcomes that were essential for the survival of the skin flap. Overall, the quality of the 20 studies included was moderate. In the present study, stem cell-based therapy effectively promotes early angiogenesis by upregulating VEGF and ultimately improving the survival rate of the skin flap. Angiogenesis in skin flaps is an intricate process involving the coordination of various cells and cytokines [12]. Rong et al. reported that human fetal skin-derived stem cell secretome promotes skin healing by activating the expression of specific genes related to angiogenesis, such as VEGF and placental growth factor (PLGF) [53]. Studies have indicated that exosomes secreted by mesenchymal stem cells may carry complex biological information, including mRNA and soluble proteins [54]. In addition, antler stem cells have been proven to stimulate fibrogenesis and angiogenesis to accelerate wound healing [55].

We found obvious heterogeneity in meta-analysis, so we further explored different research designs, including types of skin flap, area of skin flap, cell type, cell number, transplant types, and method of administration of stem cell used in treatment. However, the results may contribute to the future clinical transformation of stem cells to the bedside. Although different administration routes, cell types, skin flap areas, and species have significant effects in the prevention of skin flap necrosis,

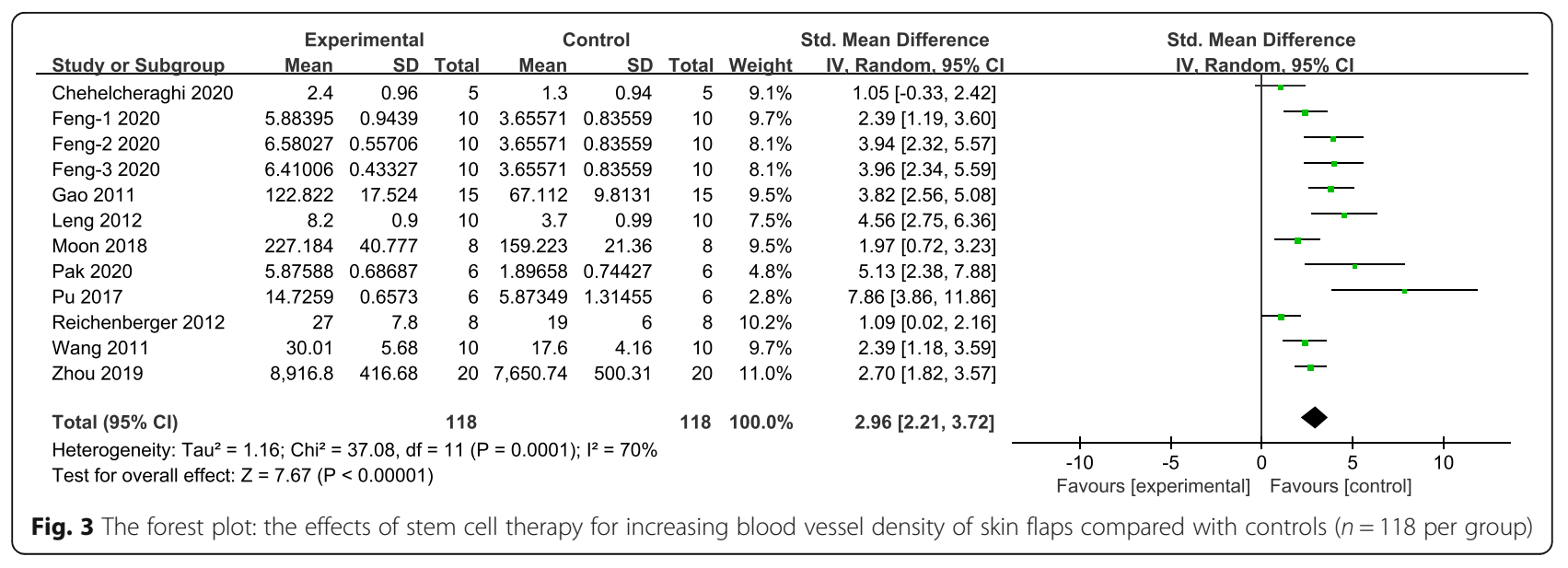




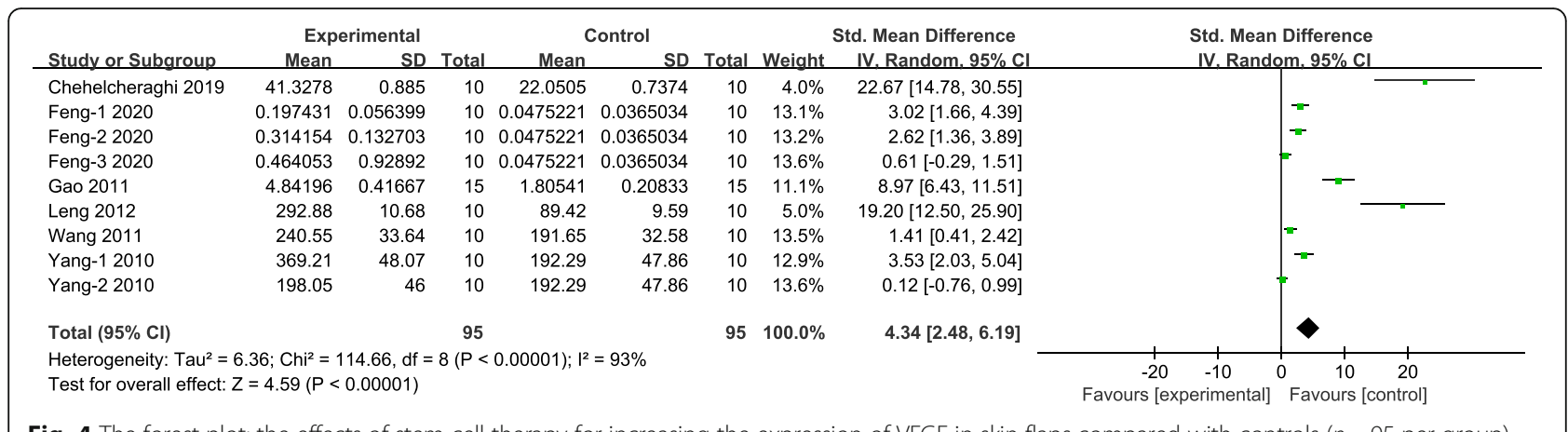

Fig. 4 The forest plot: the effects of stem cell therapy for increasing the expression of VEGF in skin flaps compared with controls $(n=95$ per group)

this study proves that stem cells are promising candidates for the prevention of skin flap necrosis.

At present, compared with MNCs (4.2\% of all groups), MSCs (50\%) and ASCs (45.8\%) are the most frequently used. Our analysis pointed out that both adipocyte and mesenchymal stem cells showed comparable efficacy in terms of skin flap survival. MNCs are a group of cells composed of multiple progenitor cells/stem cells and other cell types. As it is abundant in the peripheral blood, it can be directly collected and applied to skin

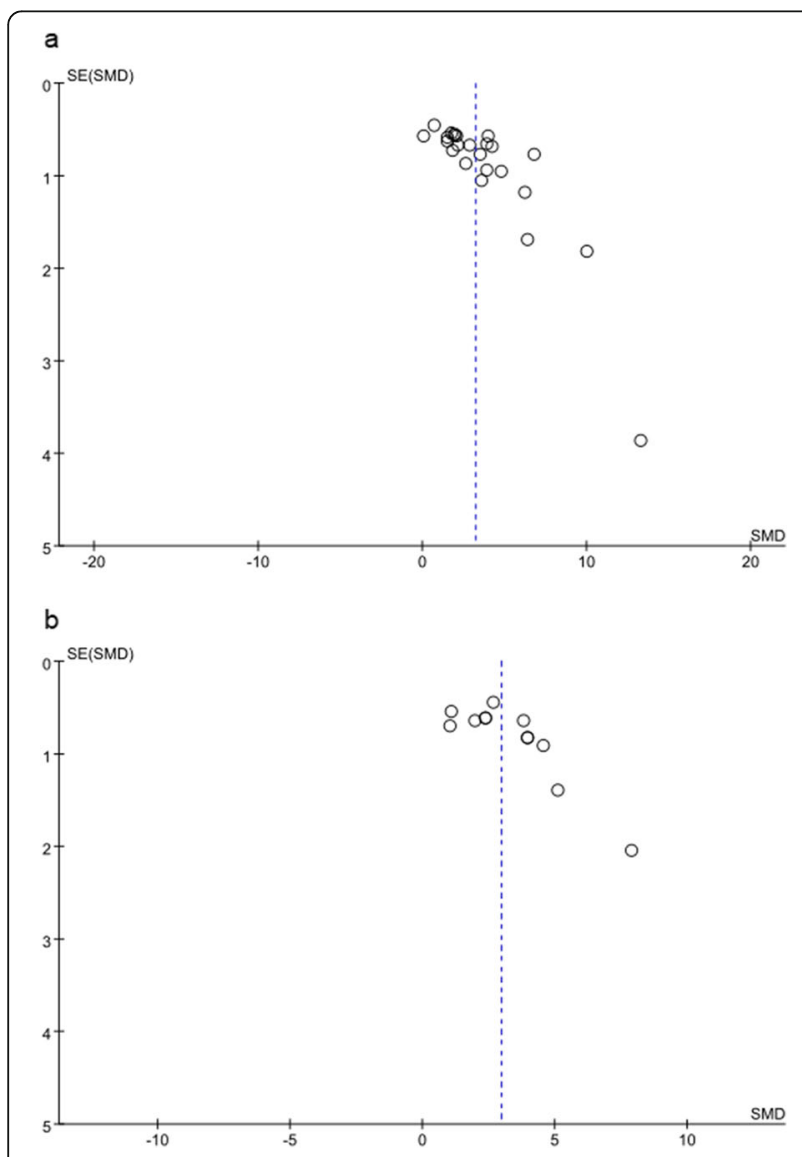

Fig. 5 Funnel plot of included studies for a survival rate of flap and b blood vessel density flap treatment. Similar to skin flaps, MNCs were also found to promote local capillary regeneration and vascular revascularization in infarcted limbs [56]. However, the effectiveness of monocytes for skin flap is significantly lower than that of the two cells mentioned above, and this result may also be affected by the limitation of only one study using such cells. Interestingly, Yang et al. [33] believed that the effectiveness of MNC transplantation in accelerating neovascularization of skin flaps may be related to basic fibroblast growth factor (bFGF) and VEGF secreted by MNCs. In clinical practice, MNCs have some characteristics, such as high safety without troublesome in vitro culture, as well as easy to isolate and obtain, which makes them have a great prospect in future research and application. However, there is growing evidence that the therapeutic mechanism of MSCs and ASCs is not only realized through paracrine cytokines and growth factors, but also can directly differentiate into vascular endothelial cells and skin components, thereby achieving vascular regeneration more effectively the goal of [57-60]. Although the most suitable type of stem cells for the clinical treatment of skin flaps cannot be directly concluded, preclinical evidence shows that MSCs and ASCs have better therapeutic effects with more trouble in their preparation process compared with MNCs. We also explored the effects of different transplant types of stem cells (Fig. S6). The subgroup analysis of transplant types showed that the efficacy of xenogeneic stem cells is significantly better than that of allogeneic stem cells applied to skin flaps (SMD 4.51 vs 2.35; Fig. S6 Supplementary materials). In recent years, the safety and effectiveness of human-derived stem cells used in clinical and preclinical studies have been confirmed [61]. This result seems to imply that HLAmatched stem cells from the donor-recipient may not be necessary to prevent skin flap necrosis.

The area of the skin flap also contributes to partial heterogeneity. In the subgroup analysis of skin flap area, both small area $\left(<10 \mathrm{~cm}^{2}\right)$ and large area $\left(\geq 10 \mathrm{~cm}^{2}\right)$ demonstrated the effect of stem cell therapy on skin flaps, with the former being more effective (SMD 3.88 vs 
2.79). That patients with more areas of skin flap usually require more nutrition for revascularization may explain this result. Besides, the larger the area of the skin flap is the greater the probability of infection, which will eventually lead to a larger area of necrosis. The most important thing is that the regenerative capacity of stem cells may have certain limitations, which makes the skin flap with a large area unable to survive more within the range of stem cell regeneration capacity. This result leads to a better effectiveness occurring on a smaller area of the skin flap. Consistent with our finding, a previous meta-analysis concluded that in the application of stem cells to treat burn wounds, smaller burn wounds are more likely to heal effectively [62]. Overall, the application of stem cells to skin flaps has shown considerable efficacy regardless of the area of the flap. It is foreseeable that stem cells can be considered as a promising treatment method for large-scale skin flaps in the clinic.

In addition, the delivery route was found to contribute to heterogeneity, accounting for $51.7 \%$. In our included studies, $25 \%$ of the groups used vascular cell delivery (including intra-arterial injection [44] and intravenous injection [35-37]), and 75\% of the groups used subcutaneous injection. In the method of administration subgroup analysis, both subcutaneous injection and intravascular injection showed the effect of stem cell therapy on skin flap, especially intra-arterial injection. Feng et al. [44] reported a significant reduction in the necrotic area of flaps after intra-arterial injection of ASCs; however, this result may be related to the axial type of skin flap used in this study. Considering that stem cell therapy aims to improve the survival rate of skin flap by promoting early angiogenesis, intra-arterial injection seems to be a reasonable and effective delivery route. We should also note that intra-arterial injection can cause vascular injury and other complications due to its invasiveness, which should be paid attention to in the future clinical operation. Although subcutaneous, intravenous, and intraarterial injections are commonly used in clinical practice, the best administration method is not yet known for sure due to the small number of studies using intravascular injection.

\section{Methodological considerations}

The ultimate goal of preclinical research is to enrich our understanding of the causes and treatments of diseases and to lay the foundation for future clinical trials [63]. Significant research results depend on accurate preclinical research reports, while the defects in experimental design lead to low-quality reports that may exaggerate or weaken the effect sizes [64]. We put forward some views on preclinical studies to prevent unsuccessful translation. First, the studies we included did not use animals with comorbidities such as diabetes, hypertension, or vascular disease. In clinical practice, most people who need flap surgery are patients with these comorbidities. The selection of inappropriate animal models may lead to inconsistent results, limiting the development of preclinical research into clinical trials [65].

Second, enough attention should be paid to the quality of the methodology. In many fields, animal reports about the results of biomedical research are inadequate [66]. Effective research results depend on accurate preclinical research reports, while insufficient experimental design will lead to low-quality reports that may lead to exaggeration or neglect of the effect [64]. Since most of the published studies based on skin flaps treated with stem cells do not use blinding of investigators and outcome assessors, the overall quality of the research is not high. To improve the overall quality of future research, we recommend that the application of stem cells in skin flap related research should be double-blind. The quality of methodology was moderate in our included studies, so we suggest that ARRIVE guidelines should be referred to for further animal experiments and design [66].

\section{Limitations}

Despite many advantages in our meta-analysis and systematic review, some potential limitations still existed and should to be considered when using the results. First of all, although our analysis does prove the significant efficacy of stem cell-based treatments on skin flaps, the heterogeneity between studies has to be mentioned. Therefore, we deal with substantial heterogeneity in the following aspects: (1) a random-effect model was used, (2) standardized mean difference was applied for all measurement outcomes, and (3) sensitivity analysis and subgroup analysis were performed to explore the sources of heterogeneity. Further subgroup analyses are needed to determine the most suitable source of stem cells, the appropriate method of administration, and the optimal type of transplantation. However, this analysis method will lead to a sharp reduction in the number of studies in each group, such as studies using intravenous injection and intra-arterial injection. More studies in the future can make the results of this study more stable and reliable. Secondly, most of the data is not easily obtained in digital form, but is extracted from graphs in published articles. The accuracy of the data will be affected by the distortion of the picture, but similarly, all groups will receive the same impact. Thirdly, we focused on the survival of skin flap as the primary outcome of our metaanalysis, with blood vessel density and expression of VEGF as the second outcome. Whether there are other essential mechanisms in the treatment of skin flap based on stem cells in addition to promoting the angiogenesis of skin flap still needs to be investigated. For example, cutaneous appendages in skin flaps should be further 
studied in future research. As cutaneous appendages including sebaceous glands and sweat glands play an important role in the skin function. Finally, possible publication bias was found in our study according to the qualitative results of funnel plots. Negative studies that were difficult to publish may contribute to publication bias, which may exaggerate the validity of the system evaluation.

\section{Conclusions}

In conclusion, the preclinical evidence of our systematic review indicates that stem cell-based therapy is effective for promoting early angiogenesis by upregulating VEGF and ultimately improving the survival rate of the skin flap. We also found that preclinical data are significantly heterogeneous, and clinical application of stem cells is rarely explored, which makes the results need more exploration. However, the differences in this study are likely to contribute to the future clinical application of stem cells and have significant guidance for future translational and research projects. In summary, small area skin flap, the administration method of intra-arterial injection, ASCs and MSCs, and xenogenic stem cells from humans showed more effective for the survival of animal skin flaps. Besides, the quality of methodology and appropriate model selection should be paid more attention to in future research. In general, stem cell-based therapy may be a promising method to prevent skin flap necrosis.

\section{Supplementary Information}

The online version contains supplementary material available at https://doi. org/10.1186/s13287-020-02103-W.

\section{Additional file 1. PRISMA checklist for minimum set of items for} reporting in systematic reviews and meta-analyses.

Additional file 2: Figure S1. Subgroup analyses of type of skin flap regarding stem cell therapy in animal model of skin flap for the primary outcome of survival rate of flap.

Additional file 3: Figure S2. Subgroup analyses of cell number regarding stem cell therapy in animal model of skin flap for the primary outcome of survival rate of flap.

Additional file 4: Figure S3. Subgroup analyses of treatment methods in the control group regarding stem cell therapy in animal model of skin flap for the primary outcome of survival rate of flap.

Additional file 5: Figure S4. Subgroup analyses of cell type regarding stem cell therapy in animal model of skin flap for the primary outcome of survival rate of flap.

Additional file 6: Figure S5. Subgroup analyses of area of skin flap regarding stem cell therapy in animal model of skin flap for the primary outcome of survival rate of flap.

Additional file 7: Figure S6. Subgroup analyses of transplant types regarding stem cell therapy in animal model of skin flap for the primary outcome of survival rate of flap.

Additional file 8: Figure S7. Subgroup analyses of method of administration of stem cells regarding stem cell therapy in animal model of skin flap for the primary outcome of survival rate of flap.

\section{Abbreviations}

ASCs: Adipose-derived stem cells; bFGF: Basic fibroblast growth factor; Cl: Confidence interval; ICR: Imprinting control region; MNCs: Mononuclear cells; MSCs: Mesenchymal stem cells; NA: Not available; PBS: Phosphatebuffered saline; PLGF: Placental growth factor; SD: Sprague Dawley; SMD: Standard mean difference; VEGF: Vascular endothelial growth factor

\section{Acknowledgements}

Not applicable.

\section{Authors' contributions}

$\mathrm{CL}, \mathrm{YL}, \mathrm{QLJ}, \mathrm{LVDM}$, and DHL designed the study. YL, QLJ, and DHL collected the data and performed all analysis. YL and LVDM wrote the manuscript. The authors read and approved the final manuscript.

\section{Funding}

This work was supported by the grant of the Key Science and Technology Program of Zhejiang Province (Grant No. 2017C01054) and the Key Science and Technology Program of Wenzhou (Grant No. ZY2019003).

\section{Availability of data and materials}

The data supporting the conclusions of this article are all online.

Ethics approval and consent to participate

Not applicable.

\section{Consent for publication}

Not applicable.

\section{Competing interests}

The authors declare that they have no competing interests.

\section{Author details}

${ }^{1}$ Department of Burn, The First Affiliated Hospital of Wenzhou Medical University, Nan Bai Xiang, Wenzhou, Zhejiang 325000, People's Republic of China. ${ }^{2}$ School of International Studies, Wenzhou Medical University, Wenzhou, Zhejiang 325000, People's Republic of China.

Received: 14 November 2020 Accepted: 14 December 2020 Published online: 07 January 2021

\section{References}

1. Basu G, Downey H, Guo S, et al. Prevention of distal flap necrosis in a rat random skin flap model by gene electro transfer delivering VEGF(165) plasmid. J Gene Medicine. 2014;16:55-65.

2. Zeltzer AA, Van Landuyt K. Reconstruction of a massive lower limb softtissue defect by giant free DIEAP flap. J Plastic Reconstructive Aesthetic Surg. 2012;65(2):e42-5.

3. Qing L, Wu P, Yu F, Zhou Z, Tang J. Use of dual-skin paddle anterolateral thigh perforator flaps in the reconstruction of complex defect of the foot and ankle. J Plastic Reconstructive Aesthetic Surg. 2018;71(9):1231-8.

4. Myers MB, Cherry G. Causes of necrosis in pedicle flaps. Plast Reconstr Surg. 1968:42(1):43-50.

5. Zhou $\mathrm{KL}$, Zhang $\mathrm{YH}$, Lin DS, Tao XY, Xu HZ. Effects of calcitriol on random skin flap survival in rats. Sci Rep. 2016;6:18945.

6. Lin R, Lin J, Li S, et al. Effects of the traditional Chinese medicine baicalein on the viability of random pattern skin flaps in rats. Drug Des Devel Ther. 2018;12:2267-76.

7. van den Heuvel MG, Buurman WA, Bast A, van der Hulst RR. Review: ischaemia-reperfusion injury in flap surgery. J Plastic Reconstructive Aesthetic Surg. 2009;62(6):721-6.

8. Chehelcheraghi F, Chien S, Bayat M. Mesenchymal stem cells improve survival in ischemic diabetic random skin flap via increased angiogenesis and VEGF expression. J Cell Biochem. 2019;120(10):17491-9.

9. Silva JJ, Pompeu DG, Ximenes NC, et al. Effects of Kaurenoic acid and arginine on random skin flap oxidative stress, inflammation, and cytokines in rats. Aesthet Plast Surg. 2015;39(6):971-7.

10. Deheng $C$, Kailiang Z, Weidong W, et al. Salidroside promotes random skin flap survival in rats by enhancing angiogenesis and inhibiting apoptosis. J Reconstr Microsurg. 2016;32(8):580-6. 
11. Aral M, Tuncer S, Şencan A, Elmas Ç, Ayhan S. The effect of thrombolytic, anticoagulant, and vasodilator agents on the survival of random pattern skin flap. J Reconstr Microsurg. 2015;31(7):487-92.

12. Sheng L, Yang M, Li H, Du Z, Yang Y, Li Q. Transplantation of adipose stromal cells promotes neovascularization of random skin flaps. Tohoku J Exp Med. 2011;224(3):229-34.

13. Mimeault M, Hauke R, Batra SK. Stem cells: a revolution in therapeuticsrecent advances in stem cell biology and their therapeutic applications in regenerative medicine and cancer therapies. Clin Pharmacol Ther. 2007; 82(3):252-64.

14. Slack JM. Origin of stem cells in organogenesis. Science (New York, N.Y.) 2008;322(5907):1498-501.

15. Chen YT, Sun CK, Lin YC, et al. Adipose-derived mesenchymal stem cell protects kidneys against ischemia-reperfusion injury through suppressing oxidative stress and inflammatory reaction. J Transl Med. 2011;9:51.

16. Yip HK, Chang LT, Wu CJ, et al. Autologous bone marrow-derived mononuclear cell therapy prevents the damage of viable myocardium and improves rat heart function following acute anterior myocardial infarction. Circulation J. 2008;72(8):1336-45.

17. Leu S, Lin YC, Yuen CM, et al. Adipose-derived mesenchymal stem cells markedly attenuate brain infarct size and improve neurological function in rats. J Transl Med. 2010;8:63.

18. Zhou F, Zhang $L$, Chen $L$, et al. Prevascularized mesenchymal stem cellsheets increase survival of random skin flaps in a nude mouse model. Am J Transl Res. 2019;11(3):1403-16.

19. Pu CM, Liu CW, Liang CJ, et al. Adipose-derived stem cells protect skin flaps against ischemia/reperfusion injury via IL-6 expression. J Investigative Dermatol. 2017;137(6):1353-62

20. Olesen AE, Andresen T, Staahl C, Drewes AM. Human experimental pain models for assessing the therapeutic efficacy of analgesic drugs. Pharmacol Rev. 2012;64(3):722-79.

21. Moher D, Liberati A, Tetzlaff J, Altman DG. Preferred reporting items for systematic reviews and meta-analyses: the PRISMA statement. Int J Surg. 2010;8(5):336-41.

22. Zhang FG, Tang XF. New advances in the mesenchymal stem cells therapy against skin flaps necrosis. World J Stem Cells. 2014;6(4):491-6.

23. Hu X, Yi Y, Zhu Y, et al. Effect of adipose-derived stem cell derived exosomes on angiogenesis after skin flap transplantation in rats. Chinese J Reparative Reconstructive Surg. 2019;33(12):1560-5.

24. Yi CG, Guo SZ, Zhang LX, et al. Promotion of the survival of ischemic skin flap by transplanted endothelial progenitor cells transfected with VEGF165 gene: an experimental study with mice. Zhonghua Yi Xue Za Zhi. 2005; 85(7):473-8.

25. Zheng $Y$, Yi C, Xia W, et al. Mesenchymal stem cells transduced by vascular endothelial growth factor gene for ischemic random skin flaps. Plast Reconstr Surg. 2008;121(1):59-69.

26. Reichenberger MA, Mueller W, Schäfer A, et al. Fibrin-embedded adipose derived stem cells enhance skin flap survival. Stem Cell Rev Rep. 2012;8(3): 844-53.

27. Bai $Y$, Han $Y D$, Yan $X L$, et al. Adipose mesenchymal stem cell-derived exosomes stimulated by hydrogen peroxide enhanced skin flap recovery in ischemia-reperfusion injury. Biochem Biophys Res Commun. 2018;500(2): 310-7.

28. Pak CS, Moon SY, Lee YE, Kang HJ. Therapeutic effects against tissue necrosis of remote ischemic preconditioning combined with human adipose-derived stem cells in random-pattern skin flap rat models. J Investigative Surg. 2020:1-8. https://doi.org/10.1080/08941939.2020.1795750. Online ahead of print.

29. Pu CM, Chen YC, Chen YC, et al. Interleukin- 6 from adipose-derived stem cells promotes tissue repair by the increase of cell proliferation and hair follicles in ischemia/reperfusion-treated skin flaps. Mediat Inflamm. 2019; 2019:2343867

30. Tomita K, Nishibayashi A, Yano K, Hosokawa K. Differentiated adiposederived stem cells promote reinnervation of rat skin flaps. Plastic and reconstructive surgery. Global Open. 2013;1(3):e22.

31. Lu F, Mizuno H, Uysal CA, Cai X, Ogawa R, Hyakusoku H. Improved viability of random pattern skin flaps through the use of adipose-derived stem cells. Plast Reconstr Surg. 2008;121(1):50-8.

32. Simman R, Craft C, McKinney B. Improved survival of ischemic random skin flaps through the use of bone marrow nonhematopoietic stem cells and angiogenic growth factors. Ann Plast Surg. 2005;54(5):546-52.
33. Yang M, Sheng L, Li H, Weng R, Li QF. Improvement of the skin flap survival with the bone marrow-derived mononuclear cells transplantation in a rat model. Microsurgery. 2010;30(4):275-81.

34. Wang JC, Xia L, Song XB, Wang CE, Wei FC. Transplantation of hypoxia preconditioned bone marrow mesenchymal stem cells improves survival of ultra-long random skin flap. Chin Med J. 2011;124(16):2507-11.

35. Tang YH, Pennington LA, Scordino JW, Alexander JS, Lian T. Dynamics of early stem cell recruitment in skin flaps subjected to ischemia reperfusion injury. Pathophysiol. 2016;23(3):221-8.

36. Suartz CV, Gaiba S, França JP, Aloise AC, Ferreira LM. Adipose-derived stem cells (ADSC) in the viability of a random pattern dorsal skin flap in rats. Acta Cir Bras. 2014:29(3):2-5.

37. Reichenberger MA, Heimer S, Schaefer A, et al. Adipose derived stem cells protect skin flaps against ischemia-reperfusion injury. Stem Cell Rev Rep. 2012:8(3):854-62.

38. Park IS, Chung PS, Ahn JC, Leproux A. Human adipose-derived stem cell spheroid treated with photobiomodulation irradiation accelerates tissue regeneration in mouse model of skin flap ischemia. Lasers Med Sci. 2017; 32(8):1737-46.

39. Moon JH, Rhee YH, Ahn JC, Kim B, Lee SJ, Chung PS. Enhanced survival of ischemic skin flap by combined treatment with bone marrow-derived stem cells and low-level light irradiation. Lasers Med Sci. 2018;33(1):1-9.

40. Leng X, Zhang Q, Zhai X, Chen Z. Local transplant of human umbilical cord matrix stem cells improves skin flap survival in a mouse model. Tohoku J Exp Med. 2012;227(3):191-7.

41. Leng $X$, Fan $Y$, Wang $Y$, et al. Treatment of ischemia-reperfusion injury of the skin flap using human umbilical cord mesenchymal stem cells (hUCMSCs) transfected with "F-5" gene. Med Sci Monitor. 2017;23:2751-64.

42. Han HH, Lim YM, Park SW, Lee SJ, Rhie JW, Lee JH. Improved skin flap survival in venous ischemia-reperfusion injury with the use of adiposederived stem cells. Microsurgery. 2015;35(8):645-52.

43. Gao W, Qiao X, Ma S, Cui L. Adipose-derived stem cells accelerate neovascularization in ischaemic diabetic skin flap via expression of hypoxiainducible factor-1a. J Cell Mol Med. 2011;15(12):2575-85.

44. Feng CJ, Perng CK, Lin CH, Tsai CH, Huang PH, Ma H. Intra-arterial injection of human adipose-derived stem cells improves viability of the random component of axial skin flaps in nude mice. J Plastic Reconstructive Aesthetic Surgery. 2020;73(3):598-607.

45. Ding JP, Chen B, Qian WJ, Bao SW, Zhao HY. Effect of bone marrow mesenchymal stem cells on perforator skin flap survival area in rats. $\mathrm{Br} \mathrm{J}$ Oral Maxillofac Surg. 2020;58(6):669-74

46. Chehelcheraghi $\mathrm{F}$, Eimani $\mathrm{H}$, Sadraie $\mathrm{SH}$, et al. Improved viability of random pattern skin flaps with the use of bone marrow mesenchymal-derived stem cells and chicken embryo extract. Iranian J Basic Medical Sci. 2015;18(8):764-72.

47. Chehelcheraghi F, Eimani H, Homayoonsadraie S, et al. Effects of acellular amniotic membrane matrix and bone marrow-derived mesenchymal stem cells in improving random skin flap survival in rats. Iran Red Crescent Med J. 2016;18(6):e25588.

48. Chehelcheraghi F, Bayat M, Chien S. Effect of mesenchymal stem cells and chicken embryo extract on flap viability and mast cells in rat skin flaps. J Investigative Surg. 2020;33(2):123-33.

49. Hashimoto I, Abe Y, Ishida S, et al. Development of skin flaps for reconstructive surgery: random pattern flap to perforator flap. J Medical Investigation. 2016;63:159-62.

50. King EA, Ozer K. Free skin flap coverage of the upper extremity. Hand Clin. 2014;30(2):201-9 vi.

51. Carroll WR, Esclamado RM. Ischemia/reperfusion injury in microvascular surgery. Head Neck. 2000;22(7):700-13.

52. Zuk PA, Zhu M, Mizuno $\mathrm{H}$, et al. Multilineage cells from human adipose tissue: implications for cell-based therapies. Tissue Eng. 2001;7(2):211-28.

53. Rong $X, L i$ J, Yang $Y$, Shi L, Jiang T. Human fetal skin-derived stem cell secretome enhances radiation-induced skin injury therapeutic effects by promoting angiogenesis. Stem Cell Res Ther. 2019;10(1):383.

54. Ranganath SH, Levy O, Inamdar MS, Karp JM. Harnessing the mesenchymal stem cell secretome for the treatment of cardiovascular disease. Cell Stem Cell. 2012;10(3):244-58.

55. Rong $\mathrm{X}, \mathrm{Chu} \mathrm{W}$, Zhang $\mathrm{H}$, et al. Antler stem cell-conditioned medium stimulates regenerative wound healing in rats. Stem Cell Res Ther. 2019. 10(1):326.

56. Lu C, Arai M, Misao Y, et al. Autologous bone marrow cell transplantation improves left ventricular function in rabbit hearts with cardiomyopathy via 
myocardial regeneration-unrelated mechanisms. Heart Vessel. 2006;21(3) 180-7.

57. Kim SW, Zhang HZ, Guo L, Kim JM, Kim MH. Amniotic mesenchymal stem cells enhance wound healing in diabetic NOD/SCID mice through high angiogenic and engraftment capabilities. PLoS One. 2012;7(7):e41105.

58. Wu Y, Chen L, Scott PG, Tredget EE. Mesenchymal stem cells enhance wound healing through differentiation and angiogenesis. Stem Cells. 2007; 25(10):2648-59.

59. Uysal CA, Ogawa R, Lu F, Hyakusoku H, Mizuno H. Effect of mesenchymal stem cells on skin graft to flap prefabrication: an experimental study. Ann Plast Surg. 2010;65(2):237-44.

60. Huang H, Liu J, Hao H, et al. Preferred M2 polarization by ASC-based hydrogel accelerated angiogenesis and myogenesis in volumetric muscle loss rats. Stem Cells Int. 2017;2017:2896874.

61. Prasad VK, Lucas KG, Kleiner Gl, et al. Efficacy and safety of ex vivo cultured adult human mesenchymal stem cells (Prochymal ${ }^{\top M}$ ) in pediatric patients with severe refractory acute graft-versus-host disease in a compassionate use study. Biol Blood Marrow Transplantation. 2011;17(4):534-41.

62. Li Y, Xia WD, Van der Merwe L, Dai WT, Lin C. Efficacy of stem cell therapy for burn wounds: a systematic review and meta-analysis of preclinical studies. Stem Cell Res Ther. 2020;11(1):322.

63. Begley CG, loannidis JP. Reproducibility in science: improving the standard for basic and preclinical research. Circ Res. 2015;116(1):116-26.

64. Leung V, Rousseau-Blass F, Beauchamp G, Pang DSJ. ARRIVE has not ARRI VEd: Support for the ARRIVE (Animal Research: Reporting of in vivo Experiments) guidelines does not improve the reporting quality of papers in animal welfare, analgesia or anesthesia. PLoS One. 2018;13(5):e0197882.

65. Briel M, Müller KF, Meerpohl JJ, et al. Publication bias in animal research: a systematic review protocol. Systematic reviews. 2013;2:23.

66. Kilkenny C, Browne WJ, Cuthill IC, Emerson M, Altman DG. Improving bioscience research reporting: the ARRIVE guidelines for reporting animal research. J Pharmacol Pharmacother. 2010;1 (2):94-9.

\section{Publisher's Note}

Springer Nature remains neutral with regard to jurisdictional claims in published maps and institutional affiliations. 